Research Article

\title{
Boundedness and Stability for Discrete-Time Delayed Neural Network with Complex-Valued Linear Threshold Neurons
}

\author{
Chengjun Duan ${ }^{1,2}$ and Qiankun Song ${ }^{1}$ \\ ${ }^{1}$ Department of Mathematics, Chongqing Jiaotong University, Chongqing 400074, China \\ ${ }^{2}$ College of Information Science and Engineering, Chongqing Jiaotong University, \\ Chongqing 400074, China
}

Correspondence should be addressed to Qiankun Song, qiankunsong@163.com

Received 21 April 2010; Accepted 30 June 2010

Academic Editor: Josef Diblik

Copyright (C) 2010 C. Duan and Q. Song. This is an open access article distributed under the Creative Commons Attribution License, which permits unrestricted use, distribution, and reproduction in any medium, provided the original work is properly cited.

The discrete-time delayed neural network with complex-valued linear threshold neurons is considered. By constructing appropriate Lyapunov-Krasovskii functionals and employing linear matrix inequality technique and analysis method, several new delay-dependent criteria for checking the boundedness and global exponential stability are established. Illustrated examples are also given to show the effectiveness and less conservatism of the proposed criteria.

\section{Introduction}

In the past decade, neural networks have received increasing interest owing to their applications in many areas such as signal processing, pattern recognition, associative memories, parallel computation, and optimization solvers [1]. In such applications, the qualitative analysis of the dynamical behaviors is a necessary step for the practical design of neural networks [2].

On the other hand, artificial neural networks are usually implemented by integrated circuits. In the implementation of artificial neural networks, time delay is produced by finite switching and finite propagation speed of electronic signals. During the implementation on very large-scale integrated chips, transmitting time delays will destroy the dynamical behaviors of neural networks. Hence it is a worthy work to consider the dynamical behaviors of neural networks with delays [3]. In recent years, some important results on the boundedness, convergence, global exponential stability, synchronization, state estimation, 
and passivity analysis have been reported for delayed neural networks; see [1-9] and the references theorems for some recent publications.

It should be pointed out that all of the above-mentioned literatures on the dynamical behaviors of delayed neural networks are concerned with continuous-time case. However, when implementing the continuous-time delayed neural network for computer simulation, it becomes essential to formulate a discrete-time system that is an analogue of the continuoustime delayed neural network. To some extent, the discrete-time analogue inherits the dynamical characteristics of the continuous-time delayed neural network under mild or no restriction on the discretization step-size, and also remains some functional similarity [10]. Unfortunately, as pointed out in [11], the discretization cannot preserve the dynamics of the continuous-time counterpart even for a small sampling period, and therefore there is a crucial need to study the dynamics of discrete-time neural networks. Recently, the dynamics analysis problem for discrete-time delayed neural networks and discrete-time systems with delay has been extensively studied; see [10-19] and references therein.

It is known that complex number calculus has been found useful in such areas as electrical engineering, informatics, control engineering, bioengineering, and other related

fields. It is therefore not surprising to see that complex-valued neural networks which deal with complex-valued data, complex-valued weights and neuron activation functions have also been widely studied in recent years [20,21]. Very recent, authors considered a class of discrete time recurrent neural networks with complex-valued weights and activation function [22, 23]. In [22], authors discussed the convergency for discrete-time recurrent neural networks with multivalued neurons, which have complex-valued weights and an activation function defined as a function of the argument of a weighted sum. In [23], the boundedness, global attractivity, and complete stability were investigated for discrete-time recurrent neural networks with complex-valued linear threshold neurons. However, the delay is not considered in [22,23], and the given criteria for checking the boundedness, global attractivity, and complete stability are conservatism to some extent. Therefore, it is important and necessary to further improve the results reported in [23].

Motivated by the above discussions, the objective of this paper is to study the problem on boundedness and stability of discrete-time delayed neural network with complex-valued linear threshold neurons.

\section{Model Description and Preliminaries}

In this paper, we consider the following discrete-time complex-valued neural network with time-delay:

$$
z(k+1)=A \sigma(z(k))+B \sigma(z(k-\tau))+H .
$$

Here $k$ is a nonnegative integer and $z(k)$ is a vector defined as $z(k)=\left(z_{1}(k), z_{2}(k)\right.$, $\left.\ldots, z_{N}(k)\right)^{T}$, where $z_{i}(k)$ denotes the activity of the $i$ th neuron. Further, $\sigma$ is a complex-valued function defined as

$$
\sigma(z)=\max \{0, \operatorname{Re}(z)\}+i \cdot \max \{0, \operatorname{Im}(z)\}
$$


and $\sigma(z(k))=\left(\sigma\left(z_{1}(k)\right), \sigma\left(z_{2}(k)\right), \ldots, \sigma\left(z_{N}(k)\right)\right)^{T}$. In (2.1), $H, A$, and $B$ are stated as the input vector $\left(h_{1}, h_{2}, \ldots, h_{N}\right)^{T}$, the connection weight matrix $\left(a_{i j}\right)_{N \times N}$ and the delayed connection weight matrix $\left(b_{i j}\right)_{N \times N}$, respectively, and $\tau$ denotes time-delay, which is a positive integer. The initial condition associated with model (2.1) is given by

$$
z(s)=\varphi(s), \quad s \in \mathbb{N}[-\tau, 0]
$$

Remark 2.1. When $B=0$, model (2.1) turns into the following model $[22,23]$

$$
z(k+1)=W \sigma(z(k))+H
$$

Hence, the model in $[22,23]$ is a special cases of the model in this paper.

Definition 2.2. A vector $z^{*}$ is called an equilibrium point of neural network (2.1), if it satisfies

$$
z^{*}=A \sigma\left(z^{*}\right)+B \sigma\left(z^{*}\right)+H
$$

Definition 2.3. The neural network (2.1) is called to be bounded if each of its trajectories is bounded.

Definition 2.4. The equilibrium point $z^{*}$ of the model (2.1) with the initial condition (2.3) is said to be globally exponentially stable if there exist two positive constants $M>0$ and $0<\varepsilon<1$ such that

$$
\left\|z(k)-z^{*}\right\| \leq M \varepsilon^{k} \sup _{s \in \mathbb{N}[-\tau, 0]}\left\|\varphi(s)-z^{*}\right\| .
$$

Throughout this paper, for any constant $c \in \mathbb{C}$, we denote $\operatorname{Re}^{+}(c)=\max \{0, \operatorname{Re}(c)\}$, $\operatorname{Re}^{-}(c)=\min \{0, \operatorname{Re}(c)\}, \operatorname{Im}^{+}(c)=\max \{0, \operatorname{Im}(c)\}$, and $\operatorname{Im}^{-}(c)=\min \{0, \operatorname{Im}(c)\}$. Now we give an assumption on connection weights.

(H) For each $i=1,2, \ldots, N$, there exist positive real numbers $\alpha_{i}$ satisfying

$$
\max _{1 \leq i \leq N}\left\{\frac{1}{\alpha_{i}} \sum_{j=1}^{N} \alpha_{j}\left[\operatorname{Re}^{+}\left(a_{i j}\right)+\left|\operatorname{Im}\left(a_{i j}\right)\right|\right]\right\}+\max _{1 \leq i \leq N}\left\{\frac{1}{\alpha_{i}} \sum_{j=1}^{N} \alpha_{j}\left[\operatorname{Re}^{+}\left(b_{i j}\right)+\left|\operatorname{Im}\left(b_{i j}\right)\right|\right]\right\}<1 .
$$

For presentation convenience, in the following, we denote

$$
\begin{aligned}
& r=\max _{1 \leq i \leq N}\left\{\frac{1}{\alpha_{i}} \sum_{j=1}^{N} \alpha_{j}\left[\operatorname{Re}^{+}\left(a_{i j}\right)+\left|\operatorname{Im}\left(a_{i j}\right)\right|\right]\right\}, \\
& \mu=\max _{1 \leq i \leq N}\left\{\frac{1}{\alpha_{i}} \sum_{j=1}^{N} \alpha_{j}\left[\operatorname{Re}^{+}\left(b_{i j}\right)+\left|\operatorname{Im}\left(b_{i j}\right)\right|\right]\right\} .
\end{aligned}
$$


Let us define

$$
v_{i}(k)=\frac{1}{\alpha_{i}}\left[\operatorname{Re}\left(\sigma\left(z_{i}(k)\right)\right)+\operatorname{Im}\left(\sigma\left(z_{i}(k)\right)\right)\right], \quad V(k)=\max _{1 \leq i \leq N}\left\{v_{i}(\mathrm{k})\right\}
$$

Let $\{A(n)\}$ be the sequence defined by

$$
A(n)= \begin{cases}V(n), & n=-\tau,-\tau+1, \ldots, 0, \\ \gamma A(n-1)+\mu A(n-1-\tau)+\beta, & n=1,2, \ldots\end{cases}
$$

where $\beta=\max _{1 \leq i \leq N}\left\{\left(1 / \alpha_{i}\right)\left(\operatorname{Re}^{+}\left(h_{i}\right)+\operatorname{Im}^{+}\left(h_{i}\right)\right)\right\}$.

To prove our results, the following lemma that can be found in [24] is necessary in this paper.

Lemma 2.5 (see [24]). Let $p$ be a nonzero number. Then $h_{n}=p^{n}$ is a solution of the homogeneous recurrence relation

$$
h_{n}-a_{1} h_{n-1}-a_{2} h_{n-2}-\cdots-a_{k} h_{n-k}=0 \quad\left(a_{k} \neq 0, n>k\right)
$$

with constant coefficients if and only if $p$ is a root of the polynomial equation

$$
x^{k}-a_{1} x^{k-1}-a_{2} x^{k-2}-\cdots-a_{k}=0 .
$$

If the polynomial equation has $k$ distinct roots $p_{1}, p_{2}, \ldots, p_{k}$, then

$$
h_{n}=c_{1} p_{1}^{n}+c_{2} p_{2}^{n}+\cdots+c_{k} p_{k}^{n}
$$

is the general solution of (2.11) in the following sense: no matter what initial values for $h_{1}, h_{2}, \ldots, h_{k}$ are given, there are constants $c_{1}, c_{2}, \ldots, c_{k}$ so that (2.13) is the unique sequence which satisfies both the recurrence relation (2.11) and the initial condition.

The polynomial equation (2.12) is called the characteristic equation of the recurrence relation (2.11) and its $k$ roots are the characteristic roots.

\section{The Main Results and Their Proofs}

Theorem 3.1. If the assumption (H) holds and $\mu>0$, the network (2.1) is bounded.

Proof. Let $\mathbb{R}^{+}=[0,+\infty)$. It is noted that the restriction $\left.\sigma(z)\right|_{\mathbb{R}}$ is nonnegative and nondecreasing and the restriction $\left.\sigma(z)\right|_{\mathbb{R}^{+}}=z$ if $z \in \mathbb{R}^{+}$. Consequently, we have

$$
\sigma(z) \geq z, \quad \forall z \in \mathbb{R}
$$


Moreover, it is easy to prove that

$$
\sigma(\operatorname{Re}(z))=\operatorname{Re}(\sigma(z)), \quad \sigma(\operatorname{Im}(z))=\operatorname{Im}(\sigma(z)), \quad \forall z \in \mathbb{C}
$$

From (2.1), we get that

$$
\begin{aligned}
\operatorname{Re}\left(z_{i}(k+1)\right) \leq & \sum_{j=1}^{N}\left[\operatorname{Re}^{+}\left(a_{i j}\right) \operatorname{Re}\left(\sigma\left(z_{j}(k)\right)\right)-\operatorname{Im}^{-}\left(a_{i j}\right) \operatorname{Im}\left(\sigma\left(z_{j}(k)\right)\right)\right] \\
& +\sum_{j=1}^{N}\left[\operatorname{Re}^{+}\left(b_{i j}\right) \operatorname{Re}\left(\sigma\left(z_{j}(k-\tau)\right)\right)-\operatorname{Im}^{-}\left(b_{i j}\right) \operatorname{Im}\left(\sigma\left(z_{j}(k-\tau)\right)\right)\right]+\operatorname{Re}^{+}\left(h_{i}\right), \\
\operatorname{Im}\left(z_{i}(k+1)\right) \leq & \sum_{j=1}^{N}\left[\operatorname{Re}^{+}\left(a_{i j}\right) \operatorname{Im}\left(\sigma\left(z_{j}(k)\right)\right)+\operatorname{Im}^{+}\left(a_{i j}\right) \operatorname{Re}\left(\sigma\left(z_{j}(k)\right)\right)\right] \\
& +\sum_{j=1}^{N}\left[\operatorname{Re}^{+}\left(b_{i j}\right) \operatorname{Im}\left(\sigma\left(z_{j}(k-\tau)\right)\right)+\operatorname{Im}^{+}\left(b_{i j}\right) \operatorname{Re}\left(\sigma\left(z_{j}(k-\tau)\right)\right)\right]+\operatorname{Im}^{+}\left(h_{i}\right)
\end{aligned}
$$

for $k \geq 0$. Note that $\operatorname{Re}^{+}\left(a_{i j}\right) \geq 0, \operatorname{Re}\left(\sigma\left(z_{j}(k)\right)\right) \geq 0,-\operatorname{Im}^{-}\left(a_{i j}\right) \geq 0, \operatorname{Im}\left(\sigma\left(z_{j}(k)\right)\right) \geq 0, \operatorname{Re}^{+}\left(b_{i j}\right) \geq$ $0, \operatorname{Re}\left(\sigma\left(z_{j}(k-\tau)\right)\right) \geq 0,-\operatorname{Im}^{-}\left(b_{i j}\right) \geq 0,-\operatorname{Im}^{-}\left(\sigma\left(z_{j}(k-\tau)\right)\right) \geq 0$, and $\operatorname{Re}^{+}\left(h_{i}\right) \geq 0$. Hence, based on monotonicity of $\left.\sigma(z)\right|_{\mathbb{R}}$, we have

$$
\begin{aligned}
\sigma\left(\operatorname{Re}\left(z_{i}(k+1)\right)\right) \leq & \sigma\left(\sum_{j=1}^{N}\left[\operatorname{Re}^{+}\left(a_{i j}\right) \operatorname{Re}\left(\sigma\left(z_{j}(k)\right)\right)-\operatorname{Im}^{-}\left(a_{i j}\right) \operatorname{Im}\left(\sigma\left(z_{j}(k)\right)\right)\right]\right. \\
& +\sum_{j=1}^{N}\left[\operatorname{Re}^{+}\left(b_{i j}\right) \operatorname{Re}\left(\sigma\left(z_{j}(k-\tau)\right)\right)-\operatorname{Im}^{-}\left(b_{i j}\right) \operatorname{Im}\left(\sigma\left(z_{j}(k-\tau)\right)\right)\right] \\
& \left.+\operatorname{Re}^{+}\left(h_{i}\right)\right) \\
= & \sum_{j=1}^{N}\left[\operatorname{Re}^{+}\left(a_{i j}\right) \operatorname{Re}\left(\sigma\left(z_{j}(k)\right)\right)-\operatorname{Im}^{-}\left(a_{i j}\right) \operatorname{Im}\left(\sigma\left(z_{j}(k)\right)\right)\right] \\
& +\sum_{j=1}^{N}\left[\operatorname{Re}^{+}\left(b_{i j}\right) \operatorname{Re}\left(\sigma\left(z_{j}(k-\tau)\right)\right)-\operatorname{Im}{ }^{-}\left(b_{i j}\right) \operatorname{Im}\left(\sigma\left(z_{j}(k-\tau)\right)\right)\right]+\operatorname{Re}^{+}\left(h_{i}\right) \\
\leq & \sum_{j=1}^{N}\left[\operatorname{Re}^{+}\left(a_{i j}\right) \operatorname{Re}\left(\sigma\left(z_{j}(k)\right)\right)+\left|\operatorname{Im}\left(a_{i j}\right)\right| \operatorname{Im}\left(\sigma\left(z_{j}(k)\right)\right)\right] \\
& +\sum_{j=1}^{N}\left[\operatorname{Re}^{+}\left(b_{i j}\right) \operatorname{Re}\left(\sigma\left(z_{j}(k-\tau)\right)\right)+\left|\operatorname{Im}\left(b_{i j}\right)\right| \operatorname{Im}\left(\sigma\left(z_{j}(k-\tau)\right)\right)\right]+\operatorname{Re}^{+}\left(h_{i}\right) .
\end{aligned}
$$


Similarly, we have

$$
\begin{aligned}
\sigma\left(\operatorname{Im}\left(z_{i}(k+1)\right)\right) \leq & \sum_{j=1}^{N}\left[\operatorname{Re}^{+}\left(a_{i j}\right) \operatorname{Im}\left(\sigma\left(z_{j}(k)\right)\right)+\left|\operatorname{Im}\left(a_{i j}\right)\right| \operatorname{Re}\left(\sigma\left(z_{j}(k)\right)\right)\right] \\
& +\sum_{j=1}^{N}\left[\operatorname{Re}^{+}\left(b_{i j}\right) \operatorname{Im}\left(\sigma\left(z_{j}(k-\tau)\right)\right)+\left|\operatorname{Im}\left(b_{i j}\right)\right| \operatorname{Re}\left(\sigma\left(z_{j}(k-\tau)\right)\right)\right]+\operatorname{Im}^{+}\left(h_{i}\right) .
\end{aligned}
$$

Hence,

$$
\begin{aligned}
v_{i}(k+1)= & \frac{1}{\alpha_{i}}\left[\operatorname{Re}\left(\sigma\left(z_{i}(k+1)\right)\right)+\operatorname{Im}\left(\sigma\left(z_{i}(k+1)\right)\right)\right] \\
\leq & \frac{1}{\alpha_{i}} \sum_{j=1}^{N}\left[\operatorname{Re}^{+}\left(a_{i j}\right)+\left|\operatorname{Im}\left(a_{i j}\right)\right|\right]\left[\operatorname{Re}\left(\sigma\left(z_{i}(k)\right)\right)+\operatorname{Im}\left(\sigma\left(z_{i}(k)\right)\right)\right] \\
& +\frac{1}{\alpha_{i}} \sum_{j=1}^{N}\left[\operatorname{Re}^{+}\left(b_{i j}\right)+\left|\operatorname{Im}\left(b_{i j}\right)\right|\right]\left[\operatorname{Re}\left(\sigma\left(z_{i}(k-\tau)\right)\right)+\operatorname{Im}\left(\sigma\left(z_{i}(k-\tau)\right)\right)\right] \\
& +\frac{1}{\alpha_{i}}\left[\operatorname{Re}^{+}\left(h_{i}\right)+\operatorname{Im}^{+}\left(h_{i}\right)\right] \\
= & \frac{1}{\alpha_{i}} \sum_{j=1}^{N} \alpha_{j}\left[\operatorname{Re}^{+}\left(a_{i j}\right)+\left|\operatorname{Im}\left(a_{i j}\right)\right|\right] v_{j}(k) \\
& +\frac{1}{\alpha_{i}} \sum_{j=1}^{N} \alpha_{j}\left[\operatorname{Re}^{+}\left(b_{i j}\right)+\left|\operatorname{Im}\left(b_{i j}\right)\right|\right] v_{j}(k-\tau) \\
& +\frac{1}{\alpha_{i}}\left[\operatorname{Re}^{+}\left(h_{i}\right)+\operatorname{Im}{ }^{+}\left(h_{i}\right)\right] \\
\leq & \gamma V(k)+\mu V(k-\tau)+\beta .
\end{aligned}
$$

Thus,

$$
V(k+1) \leq \gamma V(k)+\mu V(k-\tau)+\beta, \quad k \geq 0 .
$$

Now let $\{B(n)\}$ be a sequence with

$$
B(n)=A(n+1)-A(n), \quad n \geq-\tau .
$$

From the definition of $\{A(n)\}$, we have the following two equations:

$$
\begin{gathered}
A(n+1)=\gamma A(n)+\mu A(n-\tau)+\beta, \\
A(n)=\gamma A(n-1)+\mu A(n-1-\tau)+\beta,
\end{gathered}
$$


for $n \geq 1$. It follows from (3.9) that

$$
A(n+1)-A(n)=\gamma(A(n)-A(n-1))+\mu(A(n-\tau)-A(n-1-\tau))
$$

That is

$$
B(n)=\gamma B(n-1)+\mu B(n-1-\tau)
$$

for $n \geq 1$. Then the characteristic equation of the recurrence relation of (3.11) is

$$
x^{\tau+1}-\gamma x^{\tau}-\mu=0 .
$$

In the following, we will prove that (i) the roots of (3.12) are distinct and (ii) $|x|<1$ for each root $x$ of (3.12).

For (i), let $f(x)=x^{\tau+1}-\gamma x^{\tau}-\mu$. Then $f^{\prime}(x)=(\tau+1) x^{\tau}-\gamma \tau x^{\tau-1}$. It is clear that $f(x)$ and $f^{\prime}(x)$ are coprime since $\gamma \geq 0$ and $\mu>0$. Hence $f(x)$ has no multiple divisor, which means that (3.12) has $\tau+1$ distinct roots.

For (ii), assume to the contrary that there exists some $x_{0}$ such that $\left|x_{0}\right| \geq 1$. Then we have from (3.12) that

$$
\left|x_{0}\right|^{\tau+1} \leq r\left|x_{0}\right|^{\tau}+\mu
$$

Multiplying $\left|x_{0}\right|^{-\tau}$ on both sides of inequality (3.13), we can get

$$
\left|x_{0}\right| \leq \gamma+\mu\left|x_{0}\right|^{-\tau}
$$

Thus,

$$
1 \leq\left|x_{0}\right| \leq \gamma+\mu
$$

which is contrary to the assumption (H). Therefore, $|x|<1$ for any root $x$ of (3.12).

Let $p_{1}, p_{2}, \ldots, p_{\tau+1}$ be the distinct roots of (3.12); then $\left|p_{i}\right|<1(i=1,2, \ldots, \tau+1)$. From Lemma 2.5, we get that

$$
B(n)=c_{1} p_{1}^{n}+c_{2} p_{2}^{n}+\cdots+c_{\tau+1} p_{\tau+1}^{n}
$$

for $n \geq 1$, where $c_{1}, c_{1}, \ldots, c_{\tau+1}$ are constants which are uniquely determined by initial condition: $B(0), B(-1), \ldots, B(-\tau)$. 
From (3.8), we have

$$
\begin{aligned}
B(k-1) & =A(k)-A(k-1), \\
B(k-2) & =A(k-1)-A(k-1), \\
& \vdots \\
B(1) & =A(2)-A(1) .
\end{aligned}
$$

It follows that

$$
\begin{aligned}
A(k) & =B(k-1)+B(k-2)+\cdots+B(1)+A(1) \\
& =c_{1} \frac{p_{1}-p_{1}^{k}}{1-p_{1}}+c_{2} \frac{p_{2}-p_{2}^{k}}{1-p_{2}}+\cdots+c_{\tau+1} \frac{p_{\tau+1}-p_{\tau+1}^{k}}{1-p_{\tau+1}}+A(1),
\end{aligned}
$$

for $k \geq 1$. From $\left|p_{i}\right|<1(i=1,2, \ldots, \tau+1)$, we know that $\lim _{k \rightarrow \infty} p_{i}^{k}=0(i=1,2, \ldots, \tau+1)$, so

$$
\lim _{k \rightarrow \infty} A(k)=c_{1} \frac{p_{1}}{1-p_{1}}+c_{2} \frac{p_{2}}{1-p_{2}}+\cdots+c_{\tau+1} \frac{p_{\tau+1}}{1-p_{\tau+1}}+A(1)
$$

Thus, series $\{A(k)\}$ is bounded, that is, to say there exists a positive constant $M \in \mathbb{R}^{+}$such that $A(k) \leq M(k=1,2, \ldots)$.

By the definition of $A(k)$ and inequality (3.7), we know that $V(k) \leq A(k)$, for $k \geq-\tau$. It follows from the definition of $V(k)$ that

$$
\max _{1 \leq i \leq N}\left\{v_{i}(k)\right\}=V(k) \leq A(k) \leq M, \quad k=1,2, \ldots
$$

By the properties (3.1), (3.2) of function $\sigma$ and the definition of $v_{i}(k)$, we know

$$
\begin{aligned}
& \operatorname{Re}\left(z_{i}(k)\right) \leq \sigma\left(\operatorname{Re}\left(z_{i}(k)\right)\right)=\operatorname{Re}\left(\sigma\left(z_{i}(k)\right)\right) \leq \alpha_{i} v_{i}(k) \leq \alpha_{i} M, \\
& \operatorname{Im}\left(z_{i}(k)\right) \leq \sigma\left(\operatorname{Im}\left(z_{i}(k)\right)\right)=\operatorname{Im}\left(\sigma\left(z_{i}(k)\right)\right) \leq \alpha_{i} v_{i}(k) \leq \alpha_{i} M .
\end{aligned}
$$


On the other hand, we can get from (2.1) that

$$
\begin{aligned}
\operatorname{Re}\left(z_{i}(k+1)\right) \geq & \sum_{j=1}^{N}\left[\operatorname{Re}^{-}\left(a_{i j}\right) \operatorname{Re}\left(\sigma\left(z_{j}(k)\right)\right)-\operatorname{Im}^{+}\left(a_{i j}\right) \operatorname{Im}\left(\sigma\left(z_{j}(k)\right)\right)\right] \\
& +\sum_{j=1}^{N}\left[\operatorname{Re}^{-}\left(b_{i j}\right) \operatorname{Re}\left(\sigma\left(z_{j}(k-\tau)\right)\right)-\operatorname{Im}^{+}\left(b_{i j}\right) \operatorname{Im}\left(\sigma\left(z_{j}(k-\tau)\right)\right)\right]+\operatorname{Re}^{-}\left(h_{i}\right) \\
= & : m_{1}, \\
\operatorname{Im}\left(z_{i}(k+1)\right) \geq & \sum_{j=1}^{N}\left[\operatorname{Re}^{-}\left(a_{i j}\right) \operatorname{Im}\left(\sigma\left(z_{j}(k)\right)\right)+\operatorname{Im}^{-}\left(a_{i j}\right) \operatorname{Re}\left(\sigma\left(z_{j}(k)\right)\right)\right] \\
& +\sum_{j=1}^{N}\left[\operatorname{Re}^{-}\left(b_{i j}\right) \operatorname{Im}\left(\sigma\left(z_{j}(k-\tau)\right)\right)+\operatorname{Im}^{-}\left(b_{i j}\right) \operatorname{Re}\left(\sigma\left(z_{j}(k-\tau)\right)\right)\right]+\operatorname{Im}^{-}\left(h_{i}\right) \\
= & : m_{2}
\end{aligned}
$$

for $k \geq 0$. It is noted that $m_{1} \leq 0$ and $m_{2} \leq 0$, since $\operatorname{Re}^{-}(c) \leq 0, \operatorname{Im}^{+}(c) \geq 0, \operatorname{Im}^{-}(c) \leq 0$, $\operatorname{Re}(\sigma(z)) \geq 0$, and $\operatorname{Im}(\sigma(z)) \geq 0$. Thus, we have

$$
\begin{aligned}
\operatorname{Re}\left(z_{i}(k+1)\right) \geq & m_{1}+m_{2} \\
\geq & \sum_{j=1}^{N} \alpha_{j}\left(\operatorname{Re}^{-}\left(a_{i j}\right)-\left|\operatorname{Im}\left(a_{i j}\right)\right|\right) V(k) \\
& +\sum_{j=1}^{N} \alpha_{j}\left(\operatorname{Re}^{-}\left(b_{i j}\right)-\left|\operatorname{Im}\left(b_{i j}\right)\right|\right) V(k-\tau) \\
& +\operatorname{Re}^{-}\left(h_{i}\right)+\operatorname{Im}^{-}\left(h_{i}\right) \\
\geq & \sum_{j=1}^{N} \alpha_{j}\left(\operatorname{Re}^{-}\left(a_{i j}\right)-\left|\operatorname{Im}\left(a_{i j}\right)\right|\right) M \\
& +\sum_{j=1}^{N} \alpha_{j}\left(\operatorname{Re}^{-}\left(b_{i j}\right)-\left|\operatorname{Im}\left(b_{i j}\right)\right|\right) M \\
& +\operatorname{Re}^{-}\left(h_{i}\right)+\operatorname{Im}^{-}\left(h_{i}\right),
\end{aligned}
$$

where the last inequality is due to $0 \leq V(k) \leq A(k) \leq M$. Similarly, we can imply that

$$
\begin{aligned}
\operatorname{Im}\left(z_{i}(k+1)\right) \geq & \sum_{j=1}^{N} \alpha_{j}\left(\operatorname{Re}^{-}\left(a_{i j}\right)-\left|\operatorname{Im}\left(a_{i j}\right)\right|\right) M \\
& +\sum_{j=1}^{N} \alpha_{j}\left(\operatorname{Re}^{-}\left(b_{i j}\right)-\left|\operatorname{Im}\left(b_{i j}\right)\right|\right) M \\
& +\operatorname{Re}^{-}\left(h_{i}\right)+\operatorname{Im}^{-}\left(h_{i}\right) .
\end{aligned}
$$


From (3.21), (3.23), and (3.24), we know that the real part and imaginary part of $z_{i}(k)$ are both bounded, so each trajectory of network (2.1) is bounded.

Theorem 3.2. If there exist three $N \times N$ symmetric positive definite matrices $P, Q$, and $R$, two $N \times N$ positive diagonal matrices $D$ and $F$ such that the matrix

$$
W=\left(\begin{array}{cccc}
-P+Q+\left(\tau-\frac{1}{\tau}\right) R+D & \frac{1}{\tau} R & -\tau A^{*} R & -\tau B^{*} R \\
\frac{1}{\tau} R & -Q+F-\frac{1}{\tau} R & 0 & 0 \\
-\tau R A & 0 & A^{*}(P+\tau R) A-D & A^{*}(P+\tau R) B \\
-\tau R B & 0 & B^{*}(P+\tau R) A & B^{*}(P+\tau R) B-F
\end{array}\right)
$$

is a negative definite matrix, then network (2.1) is globally exponentially stable.

Proof. Let $\Omega=\left\{\varphi \mid \varphi \in C\left(N[-\tau, 0], R^{N}\right)\right\}$. For $\varphi \in \Omega$, define

$$
|\varphi|=\sup _{s \in \mathbb{N}[-\tau, 0]}\|\varphi(s)\|
$$

then $\Omega$ is a Banach space with the topology of uniform convergence. For any $\varphi, \psi \in \Omega$, let $z(k, \varphi)$ and $z(k, \psi)$ be the solutions of model (2.1) starting from $\varphi$ and $\psi$, respectively.

It follows from model (2.1) that

$z(k+1, \varphi)-z(k+1, \psi)=A(\sigma(z(k, \varphi))-\sigma(z(k, \psi)))+B(\sigma(z(k-\tau, \varphi))-\sigma(z(k-\tau, \psi)))$.

Let $y(k+1)=z(k+1, \varphi)-z(k+1, \psi)$ and $f(k)=\sigma(z(k, \varphi))-\sigma(z(k, \psi))$. Then (3.27) can be written as

$$
y(k+1)=A f(k)+B f(k-\tau) .
$$

Now we consider the following Lyapunov functional candidate for system (3.28) as

$$
V(k)=V_{1}(k)+V_{2}(k)+V_{3}(k),
$$


where

$$
\begin{gathered}
V_{1}(k)=y^{*}(k) P y(k), \\
V_{2}(k)=\sum_{i=k-\tau}^{k-1} y^{*}(i) Q y(i), \\
V_{3}(k)=\sum_{i=-\tau}^{-1} \sum_{l=k+i}^{k-1} \eta^{*}(l) R \eta(l), \\
\eta(k)=y(k+1)-y(k) .
\end{gathered}
$$

Then

$$
\begin{aligned}
\Delta V_{1}(k)= & y^{*}(k+1) P y(k+1)-y^{*}(k) P y(k) \\
= & (A f(k)+B f(k-\tau))^{*} P(A f(k)+B f(k-\tau))-y^{*}(k) P y(k) \\
= & f^{*}(k-\tau) B^{*} P A f(k)+f^{*}(k-\tau) B^{*} P B f(k-\tau)-y^{*}(k) P y(k) \\
& +f^{*}(k) A^{*} P A f(k)+f^{*}(k) A^{*} P B f(k-\tau), \\
\Delta V_{2}(k)= & y^{*}(k) Q y(k)-y^{*}(k-\tau) Q y(k-\tau), \\
\Delta V_{3}(k)= & \sum_{i=-\tau}^{-1}\left[\eta^{*}(k) R \eta(k)-\eta^{*}(k+i) R \eta(k+i)\right] \\
= & \tau \eta^{*}(k) R \eta(k)-\sum_{i=-\tau}^{-1}\left(\eta^{*}(k+i) R \eta(k+i)\right) .
\end{aligned}
$$

Therefore,

$$
\begin{aligned}
\Delta V(k) & =\Delta V_{1}(k)+\Delta V_{2}(k)+\Delta V_{3}(k) \\
& =\alpha^{*}(k) \Pi_{1} \alpha(k)-\sum_{i=-\tau}^{-1} \eta^{*}(k+i) R \eta(k+i),
\end{aligned}
$$

where

$$
\begin{gathered}
\alpha(k)=\left(y^{*}(k), y^{*}(k-\tau), f^{*}(k), f^{*}(k-\tau)\right)^{*}, \\
\Pi_{1}=\left(\begin{array}{cccc}
-P+Q+\tau R & 0 & -\tau A^{*} R & -\tau B^{*} R \\
0 & -Q & 0 & 0 \\
-\tau R A & 0 & A^{*}(P+\tau R) A & A^{*}(P+\tau R) B \\
-\tau R B & 0 & B^{*}(P+\tau R) A & B^{*}(P+\tau R) B
\end{array}\right) .
\end{gathered}
$$


It is easy to prove that

$$
\begin{aligned}
-\tau \sum_{i=-\tau}^{-1} \eta^{*}(k+i) R \eta(k+i) & \leq-\sum_{i=-\tau}^{-1} \eta^{*}(k+i) R \sum_{i=-\tau}^{-1} \eta(k+i) \\
& =\left(\begin{array}{c}
y(k) \\
y(k-\tau)
\end{array}\right)^{*}\left(\begin{array}{cc}
-R & R \\
R & -R
\end{array}\right)\left(\begin{array}{c}
y(k) \\
y(k-\tau)
\end{array}\right) .
\end{aligned}
$$

We define

$$
\Pi_{2}=\left(\begin{array}{cccc}
-P+Q+\left(\tau-\frac{1}{\tau}\right) R & \frac{1}{\tau} R & -\tau A^{*} R & -\tau B^{*} R \\
\frac{1}{\tau} R & -Q-\frac{1}{\tau} R & 0 & 0 \\
-\tau R A & 0 & A^{*}(P+\tau R) A & A^{*}(P+\tau R) B \\
-\tau R B & 0 & B^{*}(P+\tau R) A & B^{*}(P+\tau R) B
\end{array}\right)
$$

From (3.32) and (3.34), we have

$$
\Delta V(k) \leq \alpha^{*}(k) \Pi_{2} \alpha(k)
$$

By the definition of $\sigma$, we can get the following two inequations:

$$
\begin{aligned}
\left|\sigma\left(z_{i}(k, \varphi)\right)-\sigma\left(z_{i}(k-\psi)\right)\right| & \leq\left|z_{i}(k, \varphi)-z_{i}(k-\psi)\right| \\
\left|\sigma\left(z_{i}(k-\tau, \varphi)\right)-\sigma\left(z_{i}(k-\tau, \psi)\right)\right| & \leq\left|z_{i}(k-\tau, \varphi)-z_{i}(k-\tau, \psi)\right| .
\end{aligned}
$$

It is obvious that (3.37) is equivalent to

$$
\left(\begin{array}{c}
y(k) \\
f(k)
\end{array}\right)^{*}\left(\begin{array}{cc}
e_{i} e_{i}^{T} & 0 \\
0 & -e_{i} e_{i}^{T}
\end{array}\right)\left(\begin{array}{l}
y(k) \\
f(k)
\end{array}\right) \geq 0,
$$

and (3.38) is equivalent to

$$
\left(\begin{array}{c}
y(k-\tau) \\
f(k-\tau)
\end{array}\right)^{*}\left(\begin{array}{cc}
e_{i} e_{i}^{T} & 0 \\
0 & -e_{i} e_{i}^{T}
\end{array}\right)\left(\begin{array}{c}
y(k-\tau) \\
f(k-\tau)
\end{array}\right) \geq 0
$$

where $e_{i}$ is the unit column vector having 1 in $i$ th row and zeros elsewhere. 
Let $D=\operatorname{diag}\left(d_{1}, d_{2}, \ldots, d_{N}\right)$ and $F=\operatorname{diag}\left(f_{1}, f_{2}, \ldots, f_{N}\right)$, where $d_{i}, f_{i}>0$ for each $i$. It follows from (3.36), (3.39) and (3.40) that

$$
\begin{aligned}
\Delta V(k) \leq & \alpha^{*}(k) \Pi_{2} \alpha(k) \\
& +\sum_{i=1}^{N}\left(\begin{array}{l}
y(k) \\
f(k)
\end{array}\right)^{*}\left(\begin{array}{cc}
d_{i} e_{i} e_{i}^{T} & 0 \\
0 & -d_{i} e_{i} e_{i}^{T}
\end{array}\right)\left(\begin{array}{l}
y(k) \\
f(k)
\end{array}\right) \\
& +\sum_{i=1}^{N}\left(\begin{array}{l}
y(k-\tau) \\
f(k-\tau)
\end{array}\right)^{*}\left(\begin{array}{cc}
f_{i} e_{i} e_{i}^{T} & 0 \\
0 & -f_{i} e_{i} e_{i}^{T}
\end{array}\right)\left(\begin{array}{l}
y(k-\tau) \\
f(k-\tau)
\end{array}\right) \\
= & \alpha^{*}(k) \Pi_{2} \alpha(k) \\
& +\left(\begin{array}{l}
y(k) \\
f(k)
\end{array}\right)^{*}\left(\begin{array}{cc}
D & 0 \\
0 & -D
\end{array}\right)\left(\begin{array}{l}
y(k) \\
f(k)
\end{array}\right) \\
& +\left(\begin{array}{l}
y(k-\tau) \\
f(k-\tau)
\end{array}\right)^{*}\left(\begin{array}{cc}
F & 0 \\
0 & -F
\end{array}\right)\left(\begin{array}{l}
y(k-\tau) \\
f(k-\tau)
\end{array}\right) \\
= & \alpha^{*}(k) W \alpha(k) .
\end{aligned}
$$

Since $W$ is a negative definite matrix, we have

$$
\Delta V(k) \leq \lambda_{\max }(W)\|\alpha(k)\|^{2} \leq \lambda_{\max }(W)\|y(k)\|^{2} .
$$

By the similar method in [16], we can prove that network (2.1) is globally exponentially stable.

Corollary 3.3. If there exist an $N \times N$ positive diagonal matrix $D$ and three $N \times N$ positive definite matrices $P, Q$, and $R$ such that

$$
\Pi=\left(\begin{array}{ccc}
-P+Q+D & R & 0 \\
R & -Q-R & -R W \\
0 & -W^{*} R & W^{*}(P+R) W-D
\end{array}\right)
$$

is negative definite, then network (2.4) is globally exponentially stable.

Proof. Similar to the proof of Theorem 3.2, let $y(k+1)=z(k+1, \phi)-z(k+1, \psi)$ and $f(k)=$ $\sigma(z(k, \phi))-\sigma(z(k, \psi))$. Then

$$
y(k+1)=W f(k) .
$$

Now we consider the following Lyapunov functional candidate for system (3.44) as

$$
V(k)=V_{1}(k)+V_{2}(k)+V_{3}(k),
$$


where

$$
\begin{gathered}
V_{1}(k)=y^{*}(k) P y(k), \\
V_{2}(k)=y^{*}(k-1) Q y(k-1), \\
V_{3}(k)=\eta^{*}(k) R \eta(k), \\
\eta(k)=y(k)-y(k-1) .
\end{gathered}
$$

Then

$$
\begin{aligned}
\Delta V_{1} & =y^{*}(k+1) P y(k+1)-y^{*}(k) P y(k) \\
& =f^{*}(k) W^{*} P W f(k)-y^{*}(k) P y(k), \\
\Delta V_{2} & =y^{*}(k) Q y(k)-y^{*}(k-1) Q y(k-1), \\
\Delta V_{3} & =(y(k+1)-y(k))^{*} R(y(k+1)-y(k))-(y(k)-y(k-1))^{*} R(y(k)-y(k-1)) \\
& =(W f(k)-y(k))^{*} R(W f(k)-y(k))-(y(k)-y(k-1))^{*} R(y(k)-y(k-1)) .
\end{aligned}
$$

Let

$$
\begin{gathered}
\alpha(k)=\left(y^{*}(k), y^{*}(k-1), f^{*}(k)\right)^{*}, \\
\Pi_{1}=\left(\begin{array}{ccc}
-P+Q & R & 0 \\
R & -Q-R & -R W \\
0 & -W^{*} R & W^{*}(P+R) W
\end{array}\right) .
\end{gathered}
$$

Then

$$
\Delta V=\alpha^{*}(k) \Pi_{1} \alpha(k) .
$$

As the proof in Theorem 3.2, there exists a matrix $D=\operatorname{diag}\left(d_{1}, d_{2}, \ldots, d_{N}\right)$ with $d_{i}>0(i=$ $1, \ldots, N)$ such that

$$
\left(\begin{array}{l}
y(k) \\
f(k)
\end{array}\right)^{*}\left(\begin{array}{cc}
-D & 0 \\
0 & D
\end{array}\right)\left(\begin{array}{l}
y(k) \\
f(k)
\end{array}\right) \leq 0
$$

So

$$
\begin{aligned}
\Delta V & \leq \alpha^{*}(k) \Pi_{1} \alpha(k)-\left(\begin{array}{c}
y(k) \\
f(k)
\end{array}\right)^{*}\left(\begin{array}{cc}
-D & 0 \\
0 & D
\end{array}\right)\left(\begin{array}{l}
y(k) \\
f(k)
\end{array}\right) \\
& =\alpha^{*}(k) \Pi \alpha(k) .
\end{aligned}
$$


By the similar method in [16], we can prove that network (2.4) is globally exponentially stable.

Remark 3.4. It is known that the obtained criteria for checking stability of discretetime delayed neural networks depend on the mathematic technique and the constructed Lyapunov functionals or Lyapunov-Krasovskii functionals in varying degrees. Using elegant mathematic technique and constructing proper Lyapunov functionals or LyapunovKrasovskii functionals can reduce conservatism. So, establishing some less conservatism results will be in future works.

Remark 3.5. Recently, delay-fractioning approach is widely used to reduce conservatism; it is proved that it can reduce more conservatism than many previous methods due to the remaining of some useful terms [25]. In [25], the delay fractioning approach has been used to investigate the global synchronization of delayed-complex networks with stochastic disturbances, which has shown the potential of reducing conservatism. Using the delaypartitioning approach, we can also investigate the stability of discrete-time delayed neural networks; the corresponding results will appear in the near future.

\section{Examples}

Here, we present two examples to show the validity of our results.

Example 4.1. Consider a two-neuron neural network (2.1), where

$$
\begin{gathered}
A=\left[\begin{array}{cc}
0.3 & -0.1+0.1 i \\
-0.1-0.1 i & 0.3
\end{array}\right], \quad B=\left[\begin{array}{cc}
-0.1-0.5 i & -0.1+0.1 i \\
-0.1+0.1 i & -0.1-0.1 i
\end{array}\right], \\
H=\left[\begin{array}{ll}
10+i & 1+10 i
\end{array}\right]^{T}
\end{gathered}
$$

Taking $\alpha_{1}=13$, and $\alpha_{2}=12$, we can calculate $\gamma=4.9 / 12, \mu=7.7 / 13$, and $\gamma+\mu=$ 146.1/156 < 1. From Theorem 3.1, we know that the considered network (2.1) is bounded.

Furthermore, when $P=99 I, Q=45 I, R=I$, and $D=F=40 I$, the matrix $W$ in Theorem 3.2 is a negative definite matrix. From Theorem 3.2, we know that the considered network (2.1) is globally exponentially stable. In fact, we can verify that $(9.3+4.7 i, 0.2-12.8 i)^{T}$ and $(9.3+4.7 i, 0.2-12.8 i)^{T}$ are unique equilibrium points of $Z_{1}(k)$ and $Z_{2}(k)$ of the considered network (2.1), respectively. The global exponential stability of equilibrium points is further verified by the simulation given in Figures 1,2,3, and 4.

Example 4.2. Consider a two-neuron neural network (2.4), where

$$
W=\left(\begin{array}{cc}
0.3 & -0.4-0.5 i \\
0.2+0.1 i & -0.5
\end{array}\right), \quad H=\left[\begin{array}{ll}
-0.1-0.1 i-0.1+0.1 i
\end{array}\right]^{T}
$$

Obviously, we cannot find a dialogue positive definite matrix $D$ such that $D W$ is a Hermitian matrix, so it is impossible using the theorem in [23] to judge the stability of the considered network (2.4). 


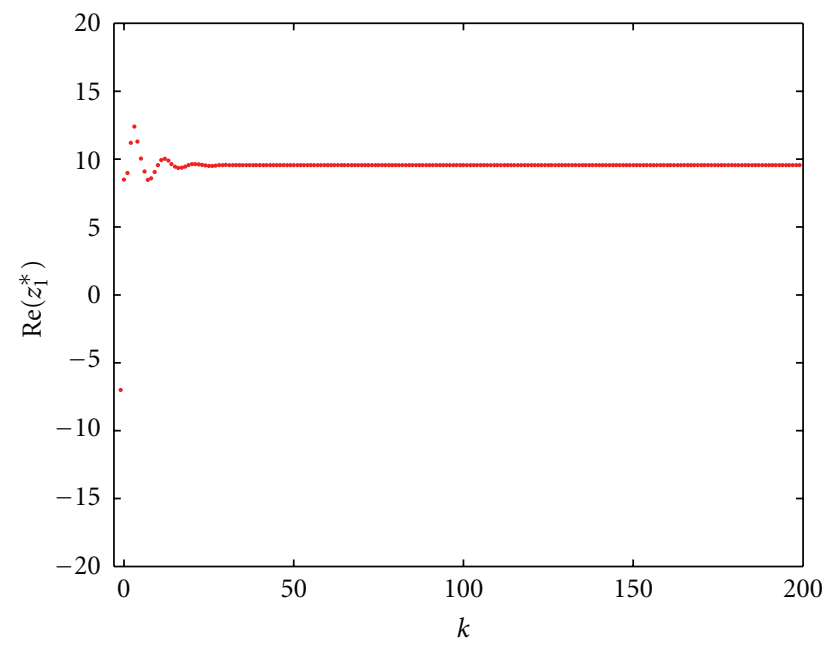

Figure 1: The behavior of the real part of $z_{1}(k)$.

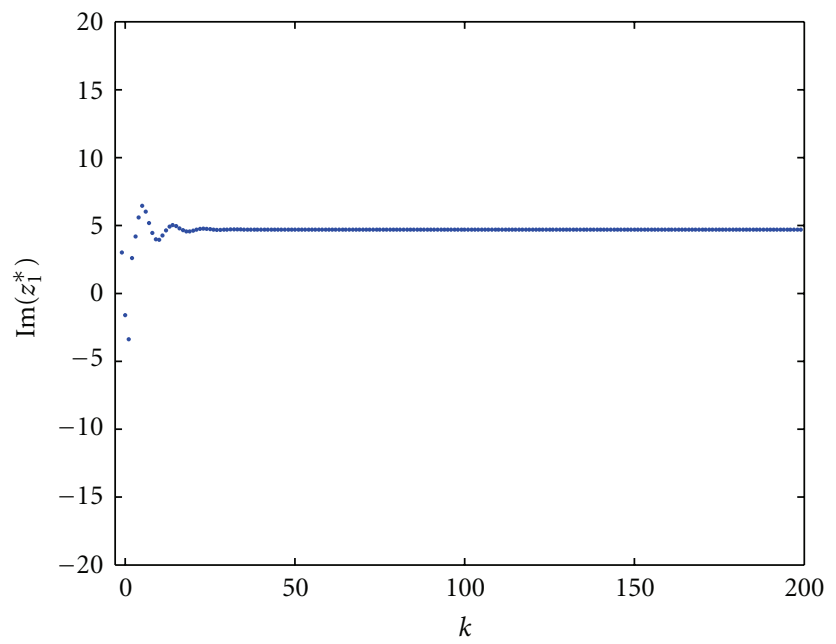

Figure 2: The behavior of the imaginary part of $z_{1}(k)$.

When $P=9 I, Q=D=4 I, R=I$, the matrix $\Pi$ in Corollary 3.3 is a negative definite matrix. From Corollary 3.3, we know that the considered network (2.4) is globally exponentially stable.

\section{Conclusion}

In this paper, the discrete-time delayed neural network with complex-valued linear threshold neurons has been considered. Several new delay-dependent criteria for checking the boundedness and global exponential stability have been established by constructing appropriate Lyapunov-Krasovskii functionals and employing linear matrix inequality 


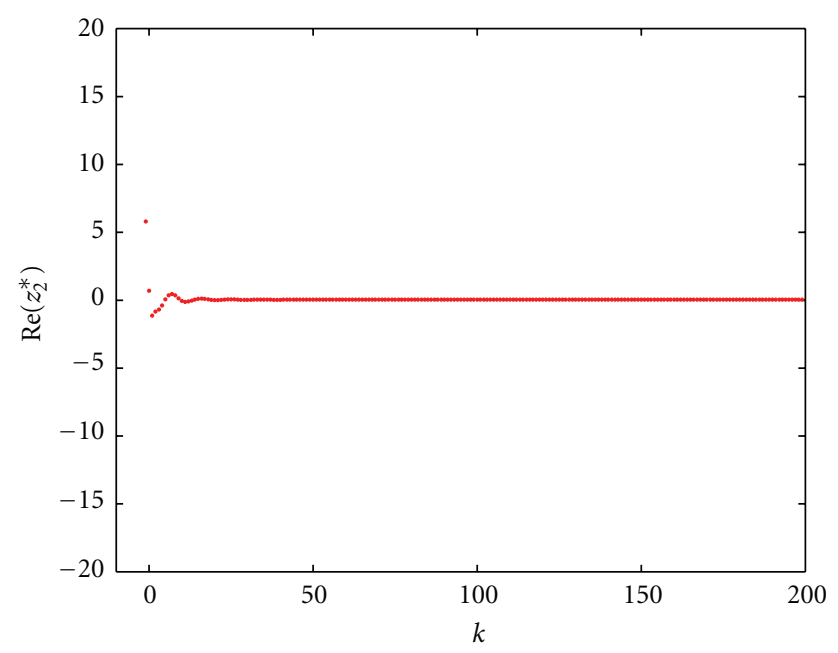

Figure 3: The behavior of the real part of $z_{2}(k)$.

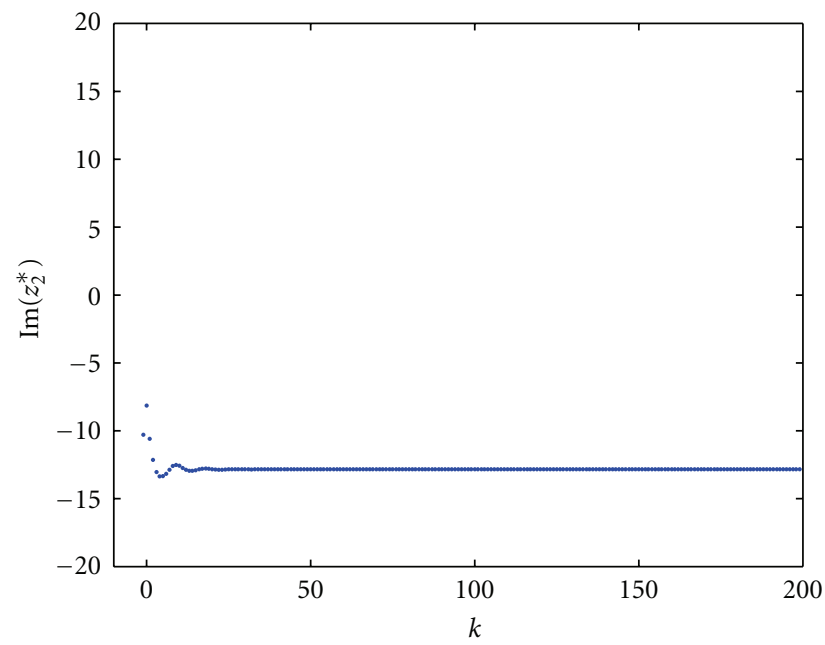

Figure 4: The behavior of the imaginary part of $z_{2}(k)$.

technique and analysis method. The proposed results are less conservative than some recently known ones in the literature, which are demonstrated via two examples.

We would like to point out that it is possible to generalize our main results to more complex systems, such as neural networks with time-varying delays $[1,8]$, neural networks with parameter uncertainties [19], stochastic perturbations [17], Markovian jumping parameters [18], and some nonlinear systems [26-32]. The corresponding results will appear in the near future. 


\section{Acknowledgment}

The work is supported by National Natural Science Foundation of China under Grants no. 60974132 and 10772152.

\section{References}

[1] S. Arik, "An analysis of exponential stability of delayed neural networks with time varying delays," Neural Networks, vol. 17, no. 7, pp. 1027-1031, 2004.

[2] T. Chen, W. Lu, and G. Chen, "Dynamical behaviors of a large class of general delayed neural networks," Neural Computation, vol. 17, no. 4, pp. 949-968, 2005.

[3] W. Lu and T. Chen, "Synchronization of coupled connected neural networks with delays," IEEE Transactions on Circuits and Systems. I. Regular Papers, vol. 51, no. 12, pp. 2491-2503, 2004.

[4] Z. Wang, Y. Liu, and X. Liu, "State estimation for jumping recurrent neural networks with discrete and distributed delays," Neural Networks, vol. 22, no. 1, pp. 41-48, 2009.

[5] S. Xu, W. X. Zheng, and Y. Zou, "Passivity analysis of neural networks with time-varying delays," IEEE Transactions on Circuits and Systems II: Express Briefs, vol. 56, no. 4, pp. 325-329, 2009.

[6] H. Jiang and Z. Teng, "Boundedness and stability for nonautonomous bidirectional associative neural networks with delay," IEEE Transactions on Circuits and Systems II: Express Briefs, vol. 51, no. 4, pp. 174180, 2004.

[7] J. Liang and J. Cao, "Boundedness and stability for recurrent neural networks with variable coefficients and time-varying delays," Physics Letters A, vol. 318, no. 1-2, pp. 53-64, 2003.

[8] J. Cao and J. Liang, "Boundedness and stability for Cohen-Grossberg neural network with timevarying delays," Journal of Mathematical Analysis and Applications, vol. 296, no. 2, pp. 665-685, 2004.

[9] H. Zhang, W. Wu, F. Liu, and M. Yao, "Boundedness and convergence of online gadient method with penalty for feedforward neural networks," IEEE Transactions on Neural Networks, vol. 20, no. 6, pp. 1050-1054, 2009.

[10] S. Mohamad and K. Gopalsamy, "Exponential stability of continuous-time and discrete-time cellular neural networks with delays," Applied Mathematics and Computation, vol. 135, no. 1, pp. 17-38, 2003.

[11] S. Hu and J. Wang, "Global robust stability of a class of discrete-time interval neural networks," IEEE Transactions on Circuits and Systems. I. Regular Papers, vol. 53, no. 1, pp. 129-138, 2006.

[12] H. Gao and T. Chen, "New results on stability of discrete-time systems with time-varying state delay," IEEE Transactions on Automatic Control, vol. 52, no. 2, pp. 328-334, 2007.

[13] M. Liu, "Global asymptotic stability analysis of discrete-time Cohen-Grossberg neural networks based on interval systems," Nonlinear Analysis: Theory, Methods \& Applications, vol. 69, no. 8, pp. 24032411, 2008.

[14] J. Yu, "New results on passivity analysis of delayed discrete-time stochastic neural networks," Discrete Dynamics in Nature and Society, vol. 2009, Article ID 139671, 17 pages, 2009.

[15] Q. Song and J. Cao, "Global dissipativity on uncertain discrete-time neural networks with timevarying delays," Discrete Dynamics in Nature and Society, vol. 2010, Article ID 810408, 19 pages, 2010.

[16] Q. Song and Z. Wang, "A delay-dependent LMI approach to dynamics analysis of discrete-time recurrent neural networks with time-varying delays," Physics Letters A, vol. 368, no. 1-2, pp. 134-145, 2007.

[17] Z. Wang, D. W. C. Ho, Y. Liu, and X. Liu, "Robust $H_{\infty}$ control for a class of nonlinear discrete timedelay stochastic systems with missing measurements," Automatica, vol. 45, no. 3, pp. 684-691, 2009.

[18] Y. Liu, Z. Wang, J. Liang, and X. Liu, "Stability and synchronization of discrete-time Markovian jumping neural networks with mixed mode-dependent time delays," IEEE Transactions on Neural Networks, vol. 20, no. 7, pp. 1102-1116, 2009.

[19] J. Liang, Z. Wang, and X. Liu, "State estimation for coupled uncertain stochastic networks with missing measurements and time-varying delays: the discrete-time case," IEEE Transactions on Neural Networks, vol. 20, no. 5, pp. 781-793, 2009.

[20] A. Hirose, Complex-Valued Neural Networks: Theories and Applications, vol. 5 of Series on Innovative Intelligence, World Scientific, River Edge, NJ, USA, 2003. 
[21] I. N. Aizenberg, N. N. Aizenberg, and J. P. L. Vandewalle, Multi-Valued and Universal Binary Neurons: Theory, Learning, Applications, Kluwer Academic Publishers, Dordrecht, The Netherlands, 2000.

[22] W. Zhou and J. M. Zurada, "A class of discrete time recurrent neural networks with multivalued neurons," Neurocomputing, vol. 72, no. 16-18, pp. 3782-3788, 2009.

[23] W. Zhou and J. M. Zurada, "Discrete-time recurrent neural networks with complex-valued linear threshold neurons," IEEE Transactions on Circuits and Systems II: Express Briefs, vol. 56, no. 8, pp. 669673, 2009.

[24] R. A. Brualdi, Introductory Combinatorics, Person Education, Upper Saddle River, NJ, USA, 2004.

[25] Y. Wang, Z. Wang, and J. Liang, "A delay fractioning approach to global synchronization of delayed complex networks with stochastic disturbances," Physics Letters A, vol. 372, no. 39, pp. 6066-6073, 2008.

[26] J. Diblík, D. Ya. Khusainov, I. V. Grytsay, and Z. Šmarda, "Stability of nonlinear autonomous quadratic discrete systems in the critical case," Discrete Dynamics in Nature and Society, vol. 2010, Article ID 539087, 23 pages, 2010.

[27] J. Baštinec, J. Diblík, and Z. Šmarda, “Existence of positive solutions of discrete linear equations with a single delay," Journal of Difference Equations and Applications, vol. 16, no. 5, pp. 1165-1177, 2010.

[28] J. Baštinec, J. Diblík, and Z. Šmarda, “Oscillation of solutions of a linear second-order discrete delayed equation," Advances in Difference Equations, vol. 2010, Article ID 693867, 12 pages, 2010.

[29] J. Diblík, D. Ya. Khusainov, and Z. Šmarda, "Construction of the general solution of planar linear discrete systems with constant coefficients and weak delay," Advances in Difference Equations, vol. 2009, Article ID 784935, 18 pages, 2009.

[30] J. Diblík, D. Ya. Khusainov, and I. V. Grytsay, "Stability investigation of nonlinear quadratic discrete dynamics systems in the critical case," Journal of Physics: Conference Series, vol. 96, no. 1, Article ID 012042, 2008.

[31] J. Baštinec and J. Diblík, "Remark on positive solutions of discrete equation $\Delta u(k+n)=-p(k) u(k)$," Nonlinear Analysis: Theory, Methods E Applications, vol. 63, no. 5-7, pp. e2145-e2151, 2005.

[32] J. Diblík, E. Schmeidel, and M. Růžičková, "Asymptotically periodic solutions of Volterra system of difference equations," Computers and Mathematics with Applications, vol. 59, no. 8, pp. 2854-2867, 2010. 


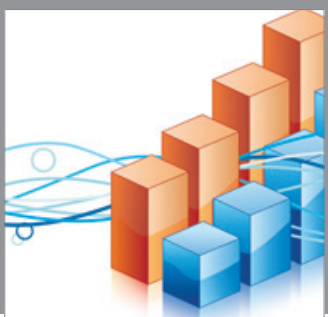

Advances in

Operations Research

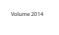

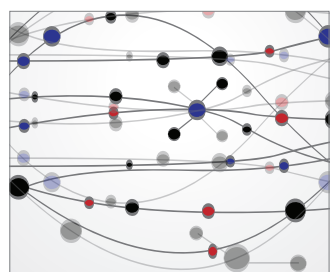

\section{The Scientific} World Journal
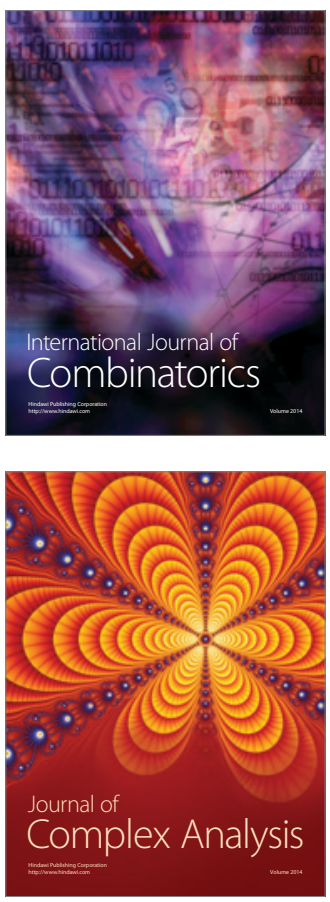

International Journal of

Mathematics and

Mathematical

Sciences
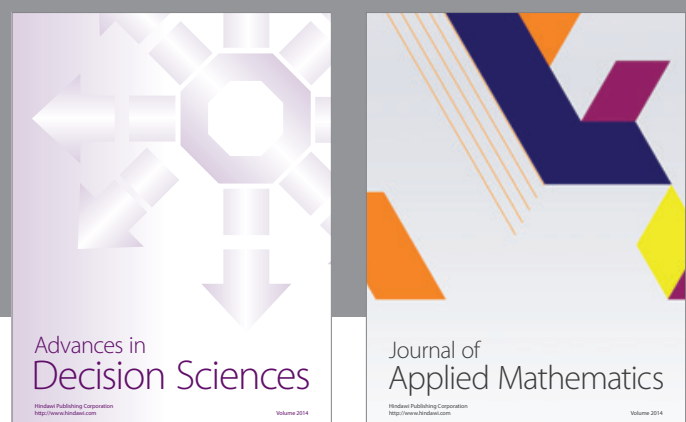

Journal of

Applied Mathematics
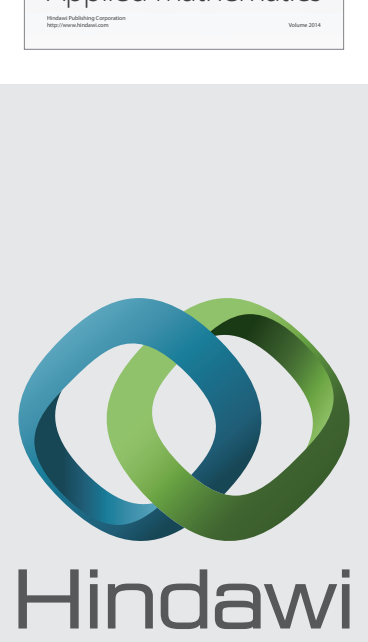

Submit your manuscripts at http://www.hindawi.com
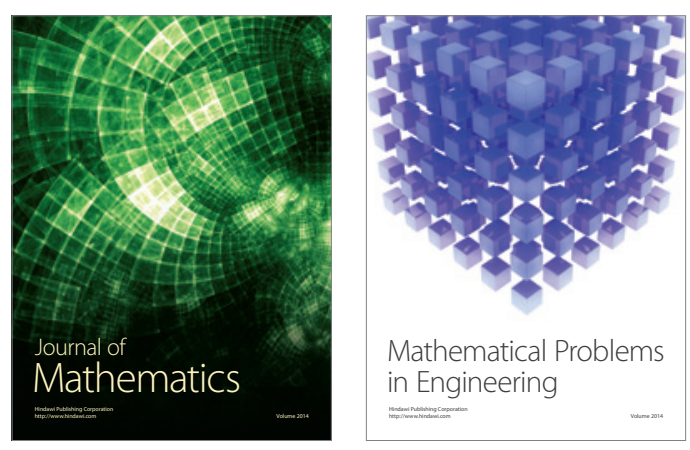

Mathematical Problems in Engineering
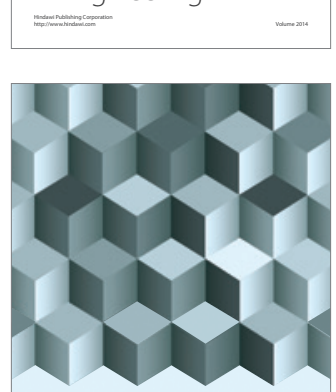

Journal of

Function Spaces
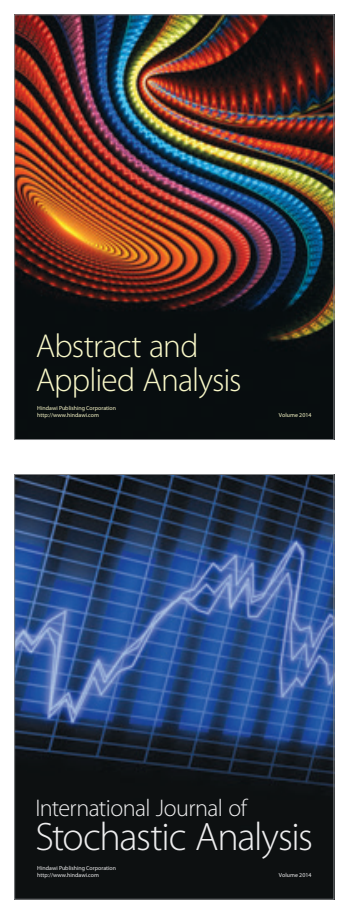

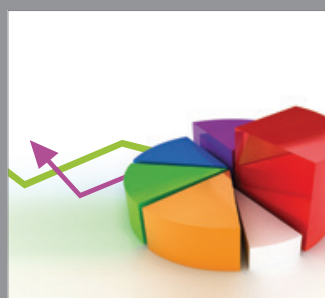

ournal of

Probability and Statistics

Promensencen
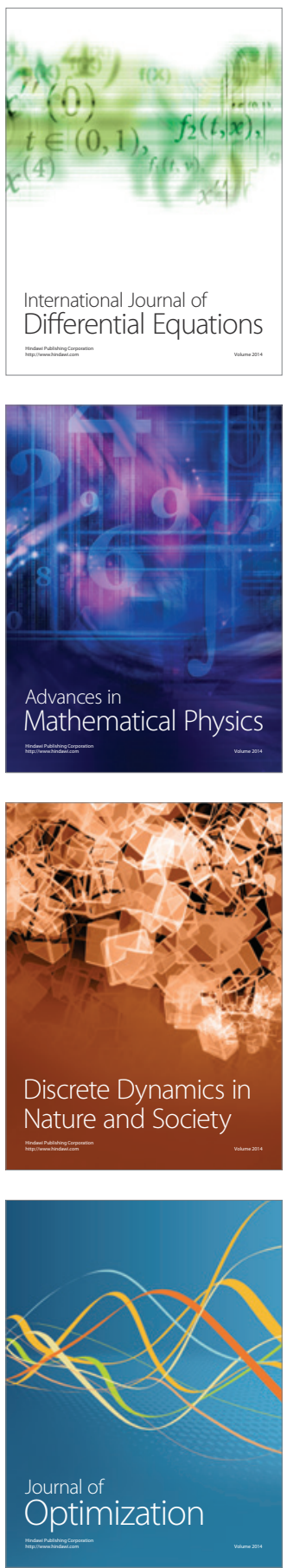